\title{
Tintas Ink-Jet para Decoracion 3D
}

\author{
FERRO SPAIN, S.A.
}

Este proyecto ha sido premiado con el Alfa de Plata en la Feria Internacional CEVISAMA VALENCIA 2011

Se han desarrollado un conjunto de diferentes tintas ink-jet, para aplicar como objeto 3D y capas extrafinas con el fin de proteger la decoración realizada mediante tecnología ink-jet. Estas nuevas tintas se obtienen a través del desarrollo de nuevas fritas basadas en estructuras vitro cristalinas monofásicas que permiten obtener efectos cerámicos mediante decoración digital. Las nuevas tintas mejoran la capacidad de aplicación mediante cabezales ink-jet con el fin de conseguir efectos estéticos y decorativos superiores a los obtenidos con la decoración convencional.

Palabras clave: decoración, tinta chorro, decoración digital, 3D.

\section{Ink-Jet for 3D Decoration}

A new set of different ink-jet inks to be apply as a 3D object and extrafine layers to protect the decoration made by ink-jet technology. These new inks are obtained through the development of new frits based on monophasic crystal vitro structures that allows ceramic effects obtained via digital decoration. The new inks improve the ability of application by ink-jet heads in order to achieve aesthetics and decorative effects than those obtained with conventional decoration.

Keywords: Ink-jet, digital decoration, frits, 3D.

\section{INTRODUCCIÓN}

La fabricación de baldosas cerámicas mediante tecnología de inyección digital está experimentando un considerable crecimiento dentro de la producción cerámica actual, debido al hecho de los ya conocidos beneficios que aporta la tecnología digital y a los cuales se suman otros dentro del proceso productivo, tanto de índole económica como medioambiental, ya que se reduce el consumo de materias primas y permite una disminución del coste de fabricación y mantenimiento de stocks, factores estos muy importantes para una gestión empresarial de éxito.

Sin embargo, la actual tecnología de inyectores, utilizados en las vigentes máquinas de decoración digital, que se emplean en la industria cerámica, demandan una nueva generación de cabezales de inyección que permitan aumentar la cantidad de tinta depositada, mayor frecuencia de disparo e incremento en la velocidad de gota.

Este nuevo tipo de cabezales deben de poder resolver las necesidades asociadas a la tecnología digital de la producción cerámica, que en resumen son resolución y cantidad de material depositado.

La respuesta a este dilema esta asociada a las siguientes preguntas:

¿Qué tipo de cabezal es necesario?

¿Qué material se va a aplicar con este cabezal?

Dado el bajo espesor de tinta aplicable con la tecnología actual de cabezales de decoración digital, hasta ahora, no había sido posible la obtención de efectos cerámicos, utilizando serigrafías y esmaltes convencionales.

Desde Ferro Spain, S.A. se ha querido abordar este reto desde el punto de vista de los materiales empleados. Asumiendo la idea de que unos cabezales de impresión que permitiesen el uso de material cerámico mas grosero abriría muchas posibilidades técnicas, estéticas, funcionales y por supuesto económicas. Como líder en el sector de fabricación de tintas digitales, este hecho se resalta en un futuro próximo como una de las líneas ineludibles que el sector cerámico va a emplear en la evolución del proceso productivo actual y que permitirá redefinir las características del sector industrial cerámico.

La nueva generación de tintas 3D aplicadas con los cabezales actuales sobre los distintos tipos de esmaltes permiten la obtención de efectos cerámicos que en algunos casos presentan mejores prestaciones técnicas y / o estéticas que las que se obtienen por medio de la decoración convencional con pantallas serigráficas y rodillos.

Hasta el momento, la decoración ink-jet se había limitado a conseguir un diseño de alta definición y variedad cromática, por lo que se pueden obtener azulejos con diseños reales pero necesitan de una segunda aplicación con otra tecnología para obtener efectos brillo-mate, protecciones, penetrantes, metálicos, etc...

Por lo tanto, si se consigue una imagen muy real, ¿Porqué no conseguir variedad de texturas con la misma máquina de inyección sin tener que necesitar otras formas de aplicación? 
El futuro inmediato pasa por una aplicación total con la máquina de inyección.

Por lo tanto, hemos iniciado dicho reto con una variedad de texturas nuevas que se han mencionado anteriormente.

\section{NUEVA GAMA DE PRODUCTOS}

Ferro Spain, S.A. ha desarrollado una serie de tintas que aúna todas las características necesarias para lograr los efectos de opacidad, maticidad, blancura, poder cubriente y protección de aplicación ink- jet.

Esta nueva serie de productos amplía las posibilidades de decoración digital al tiempo que permite nuevos diseños y funcionalidades.

Pese a que muchos de estos efectos se pueden obtener en una gran diversidad se soportes, Ferro Spain, S.A. ha trabajado en sus laboratorios de $\mathrm{I}+\mathrm{D}+\mathrm{i}$ para conseguir la optimización y potenciación de estos, con el desarrollo de composiciones de sustratos vidriados que permitan alcanzar superiores niveles de calidad.

\section{DESARROLLO EXPERIMENTAL}

En el caso de la aplicación por inyección de chorro de tinta, existen determinadas limitaciones de tamaño máximo de partícula aceptable, para la actual tecnología de impresión así como derivadas de la cantidad máxima de tinta que se puede depositar asociada a la velocidad de línea de esmaltado aplicada.

Ello se traduce en una gran dificultad en el control del tamaño de partícula, carga de sólidos, estabilización de la tinta y selección de la configuración de parámetros de impresión óptimos para obtener los resultados deseados por medio del empleo de esta tecnología.

\section{Preparación de composiciones.}

La composición de las tintas TID_3D está basada en estructuras vitrocristalinas monofásicas de alta pureza química y con un comportamiento newtoniano.

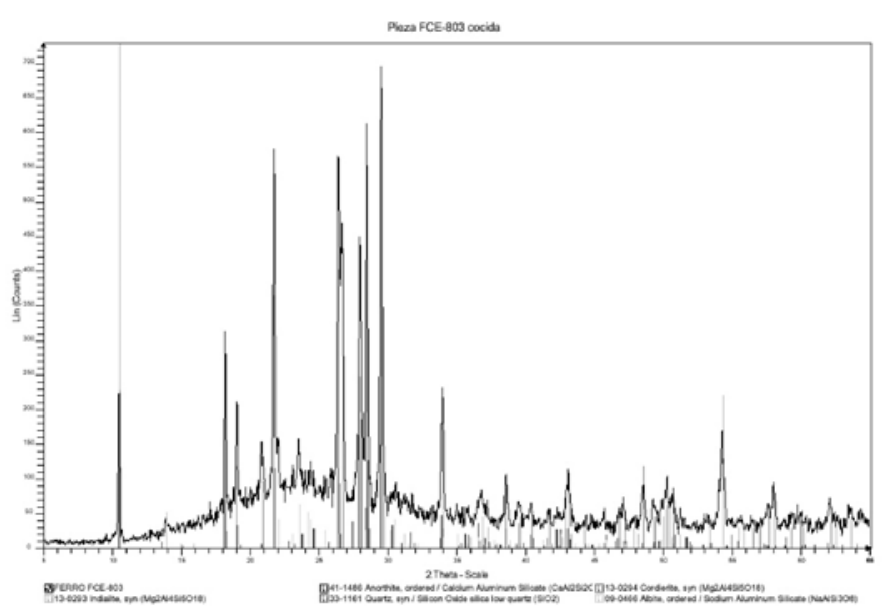

Estas estructuras cristalinas monofásicas de alta pureza han sido desarrolladas por medio de técnicas de nucleación y crecimiento de cristales en condiciones térmicas de de ciclos $>3 \mathrm{~h}$ y alta temperatura $>1500{ }^{\circ} \mathrm{C}$ ) y unas condiciones muy específicas, fusión de larga duración (3-4 horas) y alta temperatura $\left(1550-1570{ }^{\circ} \mathrm{C}\right)$.

Los procesos térmicos han sido en algunos casos por medio de la fusión en horno rotativo y en otros por medio de la calcinación en hornos caja.

Los diferentes efectos obtenidos son función de la naturaleza de la fase cristalina obtenida, así se distingue entre:

\section{Opacificantes.}

En función de la naturaleza del cristal obtenido, su índice de refracción y del tamaño y forma de los centros de dispersión obtendremos diferentes grados de opacificación.

Cumpliendo con los requisitos anteriormente citados han sido ensayados diferentes materiales que presentan una importante capacidad opacificante. Entre los que se han descartado aquellos que no son adecuados para proporcionar blancura debido al hecho que presentan color o bien que se colorean fácilmente durante el proceso cerámico.

En resumen, aprovechando las características necesarias para la aplicación digital opacificante, y teniendo en cuenta los requisitos anteriormente citados, se han obtenido una serie de tintas para la obtención del "color "blanco en la gama digital.

\section{Texturas y Protecciones.}

La modulación de la refractariedad y distribución de tamaño de partícula sumada a la adecuada selección de la composición química adecuada permite la obtención de texturas y cubiertas con suficiente resistencia química y a la abrasión, a través de la utilización de tecnología ink-jet, lo cual no se había conseguido hasta la fecha.

\section{Algunos ejemplos de estas tintas están basados en:}

\section{Cordierita $\mathrm{Sio}_{2}-\mathrm{Al}_{2} \mathrm{O}_{3}-\mathrm{MgO}$}

La cordierita, $2 \mathrm{Al}_{2} \mathrm{O}_{3.5} \mathrm{SiO}_{2.2} \mathrm{MgO}$, es un aluminosilicato de magnesio estable dentro del sistema ternario.: $\mathrm{SiO}_{2}-\mathrm{Al}_{2} \mathrm{O}_{3}-\mathrm{Mg} 0$. $\mathrm{Su}$ cristalización ocurre en una zona reducida del diagrama de fases $\mathrm{SiO}_{2}-\mathrm{Al}_{2} \mathrm{O}_{3}-\mathrm{Mg} 0$, comprendida entre cinco puntos eutécticos cuyas temperaturas varían entre $1355-1400^{\circ} \mathrm{C}$.

Sistema ortorómbico

Dureza Mohs 7-7,5

Aspecto mate.

Mullita $\mathrm{SiO}_{2}-\mathrm{Al}_{2} \mathrm{O}_{3}$

La mullita se emplea como material estructural debido a sus excelentes propiedades mecánicas aún a altas temperaturas.

Sistema cristalino estable a alta temperatura.

Dureza Mohs 6-7

Aspecto mate 


\section{Diopsido $\mathrm{SiO}_{2}-\mathrm{MgO}-\mathrm{CaO}$.}

Es un piroxeno monoclínico de fórmula $\mathrm{MgCaSi}_{2} \mathrm{O}_{6}$. Posee una serie de solución completa con la hedenbergita $\left(\mathrm{FeCaSi}_{2} \mathrm{O}_{6}\right)$.

Sistema monoclínico

Dureza Mohs 5-6

Aspecto mate.

Todas estos materiales son muy estables frente a la temperatura y aportan diferentes propiedades

\section{Efectos lustrados.}

El efecto conocido como lustre es obtenido por la capacidad que poseen determinados productos de generar estructuras que alteran la reflexión de la luz en forma de patrones especiales. Los materiales comúnmente empleados conllevan una problemática particular debido a sus elevadísimas densidades que dificultan la obtención de tintas ink-jet que puedan ser empleadas bajo el marco digital actual. La cuidada selección de materias primas junto con un procesado específico que minimiza los problemas anteriores, habilita la posibilidad de obtener este efecto a través de la tecnología digital.

\section{APLICACIÓN INDUSTRIAL}

\section{Procesado de la tinta.}

Una vez obtenidas las distintas estructuras cristalinas por medio de los correspondientes procesos térmicos se inicia la siguiente etapa de pre-molturación, en la que se procede de forma mecánica a la progresiva reducción del tamaño de partícula hasta alcanzar valores muy bajos.

Este proceso de molturación se debe de realizar en distintas etapas y medios:

Para poder pasar de granos de frita hasta un tamaño de partícula aproximado a $1 \mu \mathrm{m}$ es necesario molturar con distintos diámetros de bolas en los molinos.

En la última etapa de molturación conjunta con el medio se finaliza el proceso en el que se consigue que la tinta final tenga las propiedades reológicas adecuadas de aplicación en los cabezales.

Una vez obtenido el nuevo conjunto de tintas ink-jet, y verificado su funcionamiento en una maquina industrial a escala de laboratorio, se han elaborado piezas cerámicas tanto para revestimiento como pavimento, según los diferentes ciclos de cocción industriales.


Imágenes de algunas piezas cerámicas obtenidas tras la aplicación del nuevo conjunto de tintas ink-jet. 


\section{Interacción con el esmalte}

El paso siguiente fue conseguir según los diferentes efectos estéticos deseados, preparar la combinación apropiada de los diferentes materiales generados, con el fin de producir piezas cerámicas en las que toda la decoración haya sido realizada mediante aplicación ink-jet, de forma que se consigue una modulación de los efectos cerámicos acorde con la decoración gráfica empleada. El motivo por el cual este objetivo no se había alcanzado, es que hasta ahora no se había conseguido aplicar mediante tecnología ink-jet los diferentes efectos cerámicos: mate, lustre, blanco, textura, etc., que tal y como se obtienen en la actualidad en la totalidad de la producción cerámica industrial es mediante la aplicación con maquinas serigráficas convencionales.

En resumen, las piezas cerámicas presentadas son el resultado de la aplicación en maquinas ink-jet actuales, con la única necesidad de incrementar el número de unidades de inyección mas un nuevo conjunto de tintas ink-jet, confeccionado de forma adecuada para cumplir con requisitos predeterminados, y sintetizadas a partir de compuestos iniciales preparados expresamente para este uso.

Se ha verificado que este tipo de composiciones aplicadas de forma superficial en espesores tan bajos producen interacciones muy intensas con el esmalte, tomando de él los elementos necesarios para la formación de estructuras adaptables a la fase vítrea, sin que integren dentro de la matriz del esmalte.

De esta forma, se consigue que las zonas en las que contiene estas tintas tengan las propiedades tenaces de las fases cristalinas de partida pero también mantienen una estructura vítrea adecuada que les confiere aspecto agradable y características técnicas cerámicas.

A su vez, un requisito de estas tintas $3 D$ es que sean compatibles con las actuales tintas.

\section{Efectos tridimensionales y refuerzo técnico.}

El efecto tridimensional se consigue gracias a la interacción de la luz entre las zonas con y si aplicación como consecuencia del gran salto de índice de refracción entre ambas, no habiendo entre ellas ningún salto de nivel.

El refuerzo técnico conseguido en las zonas donde están aplicadas las tintas es extraordinario, no se consigue desgastar después de un ciclo de PEI de 12.000 rev. Siendo también resistente a todo tipo de agentes químicos.

\section{BIBLIOGRAFIA:}

1. J. L. Amorós, A. Barba, V. Beltrán, Estructuras cristalinas de los silicatos y óxidos de las materias primas cerámicas. Castellón: Instituto de Tecnología Cerámica, 1994.

2. F. H. Norton Cerámica fina. Barcelona: Omega, 1975

3. J. Konta Properties of ceramic raw materials. EN:Ceramic Monographs. Freiburg: Schmid, 1981

4. L.F. Verdeja, J.P. Sancho, A. Ballester. Materiales Refractarios y Cerámicos. Síntesis. Madrid, 2008

5. M. Kumar.Concrete, Structure, Properties, and Materials. Ed. Prentice-Hall, Inc Englewood. New Jersey 1986 